\title{
Obtaining Pentosidine Levels as an Adjunct to Determine a Patient's Candidacy for Vertebral Stabilization for a Vertebral Compression Fracture
}

\author{
Omar Viswanath ${ }^{1,2,3}$, Vwaire Orhurhu ${ }^{4}$, Ivan Urits ${ }^{4}$ \\ ${ }^{1}$ Valley Anesthesiology and Pain Consultants, Phoenix, AZ, USA \\ ${ }^{2}$ Department of Anesthesiology, University of Arizona College of Medicine-Phoenix, Phoenix, AZ, USA \\ ${ }^{3}$ Department of Anesthesiology, Creighton University School of Medicine, Omaha, NE, USA \\ ${ }^{4}$ Department of Anesthesia, Critical Care, and Pain Medicine, Beth Israel Deaconess Medical Center, Harvard Medical School, Boston, MA, USA
}

We read the clinical study by Choi et al. [1], "Feasibility of serum pentosidine level as a potential risk factor for osteoporotic vertebral compression fracture," with great interest.

"There was a statistically significant difference in the mean serum pentosidine level $(p=0.04)$ of the vertebral fracture group $(110.8 \mathrm{ng} / \mathrm{mL})$ and the non-fracture group $(64.3 \mathrm{ng} / \mathrm{mL})$. Logistic regression analyses showed that serum pentosidine was significantly associated with osteoporotic vertebral compression fracture. These results suggest that increased serum pentosidine level could be a potential marker for osteoporotic vertebral compression fracture."[1]

Pentosidine is a well-known advanced glycation end product that was discovered from aging human extracellular matrix [2]. Pentosidine deteriorates the osteoblastic function in the bone [3]. "Our results suggest that increased serum pentosidine level could be a potential risk factor for osteoporotic vertebral compression fracture [1]."

The authors' study conclusion brings to the light that pentosidine may also a valuable adjunct lab test in the workup for patients with acute osteoporotic vertebral compression fracture to see if they are candidates for a percutaneous vertebral stabilization procedure such as a vertebroplasty or kyphoplasty. The objective of these procedures are to consolidate the fracture and restore the height of the vertebral body to reduce vertebral and regional kyphosis. Stabilization of the acute vertebral body fracture leads to a reduction in pain and thus restores the spinal support function as quickly as possible, which is particularly important in the elderly population [4].

In the workup for patients with osteoporotic vertebral compression fracture, magnetic resonance imaging (MRI) is the study of choice. MRI can demonstrate marrow edema, accentuated on short-TI inversion recovery or fat-saturated T2-weighted sequences, and can thus distinguish acute and unhealed fractures from healed fractures. Fractures with little height loss or kyphosis may become apparent only on MRI [5].

Obtaining a serum pentosidine that shows an elevate level can provide an additional confirmation that substantiates the history, physical exam, and MRI. In addition there are a certain subset of patients where it may be difficult to determine the chronicity of the fracture on MRI

Received Dec 12, 2018; Accepted Dec 20, 2018

Corresponding author: Omar Viswanath

Valley Anesthesiology and Pain Consultants, 645 E. Missouri Avenue, Suite 300, Phoenix, AZ 85012, USA

Tel: +1-480-467-2273, Fax: +1-602-648-4361, E-mail: viswanoy@gmail.com 
and it is hard to determine strictly based off of imaging. In these particular situations, obtaining a pentosidine serum level that shows increased levels may provide more evidence towards possibly doing the procedure.

\section{Conflict of Interest}

No potential conflict of interest relevant to this article was reported.

\section{References}

1. Choi DH, Lee SM, Lim SA, Choi YS. Feasibility of serum pentosidine level as a potential risk factor for osteoporotic vertebral compression fracture. Asian Spine J 2018;12:992-7.
2. Sell DR, Monnier VM. Structure elucidation of a senescence cross-link from human extracellular matrix: implication of pentoses in the aging process. J Biol Chem 1989;264:21597-602.

3. Sanguineti R, Storace D, Monacelli F, Federici A, Odetti P. Pentosidine effects on human osteoblasts in vitro. Ann N Y Acad Sci 2008;1126:166-72.

4. Bousson V, Hamze B, Odri G, Funck-Brentano T, Orcel P, Laredo JD. Percutaneous vertebral augmentation techniques in osteoporotic and traumatic fractures. Semin Intervent Radiol 2018;35:309-23.

5. Benz BK, Gemery JM, McIntyre JJ, Eskey CJ. Value of immediate preprocedure magnetic resonance imaging in patients scheduled to undergo vertebroplasty or kyphoplasty. Spine (Phila Pa 1976) 2009;34:60912. 